04

\title{
Определение индикатрисы упругого рассеяния электрона на атоме из зондовых измерений функции распределения рассеянных электронов в низковольтном пучковом разряде в инертных газах
}

\author{
(C) A.C. Мустафаев, ${ }^{1}$ В.С. Сухомлинов ${ }^{1,2}$
}

${ }^{1}$ Санкт-Петербургский горный университет, 199106 Санкт-Петербург, Россия

${ }^{2}$ Санкт-Петербургский государственный университет, 198504 Санкт-Петербург, Россия

e-mail: alexmustafaev@yandex.ru,v_sukhomlinov@mail.ru

Поступило в Редакцию 22 февраля 2018г.

В окончательной редакции 10 апреля 2019г.

Принята к публикации 21 октября 2019 г.

\begin{abstract}
Предложен метод экспериментального определения индикатрисы упругого рассеяния электрона на атоме из зондовых измерений функции распределения рассеянных электронов в пролетном режиме низковольтного пучкового разряда. Решено кинетическое уравнение Больцмана методом разложения в ряд по числам столкновений, и экспериментально определена рассеянная часть функции распределения электронов методом плоского одностороннего зонда, который ранее был развит авторами для исследований пучковых разрядов. Метод опробован в плазме низковольтного пучкового разряда в Не. Получено хорошее соответствие экспериментально измеренной индикатрисы упругого рассеяния электронов с энергией $30 \mathrm{eV}$ на атомах Не и известных данных о дифференциальном сечении, в том числе полученных из пучковых экспериментов. Показано, что для реализации разработанного метода определения индикатрисы упругого рассеяния электронного пучка на атомах не требуется никаких априорных данных.
\end{abstract}

Ключевые слова: низковольтный пучковый разряд, индикатриса упругого рассеяния электрона, метод плоского одностороннего зонда, кинетическое уравнение Больцмана.

DOI: 10.21883/JTF.2020.04.49081.87-18

\section{Введение}

В работе [1] впервые была предложена модификация известного метода плоского одностороннего зонда для определения анизотропных функций распределения (ФР) электронов (ФРЭ) [2-14] и ионов (ФРИ) [1518] в газоразрядной плазме. Авторами [1] найдены условия, которым должна удовлетворять ФР по углам и энергиям, чтобы при реализации этого метода вторая производная была, с известной степенью точности, пропорциональна ФР. Это позволило экспериментально определить ФРЭ в пролетном режиме гелиевого низковольтного пучкового разряда (НПР) в области энергии первоначального пучка порядка $30 \mathrm{eV}$ в условиях, когда традиционная методика неприменима. Было показано, что ФРЭ после ускорения электронов в прикатодном ленгмюровском слое представляет собой практически монокинетический электронный газ, поскольку по данным [1] в большей части межэлектродного пространства электрическое поле мало, а в пролетном режиме длина пробега электрона относительно неупругих столкновений с атомами много больше межэлектродного расстояния. Этот газ состоит, во-первых, из нерассеянной части, которая является максвелловским распределением, сдвинутым в результате ускорения в поле катодного падения, и описывается функцией:

$$
\begin{aligned}
f^{0}(\mu, z) & =\frac{1}{\pi \mu E i\left(\frac{1}{2 \beta_{0} \mu^{2}}\right)} \exp \left(-\frac{1}{2 \beta_{0} \mu^{2}}\right) \\
& \times \exp (-z)\left[1+O\left(2 \beta_{0}\right)\right], \quad \mu \geq 0,
\end{aligned}
$$

где $T_{k}$ - температура катода, $\theta-$ угол между направлением электрического поля и скоростью электрона, $\beta_{0}=\frac{k T_{k}}{2 E_{0}} \ll 1, \mu=\cos \theta, E_{i}\left(x=\int_{x}^{\infty} \frac{\exp (-y)}{y} d y-\right.$ интегральная показательная функция, и, во-вторых, из рассеянной части $F_{s c}(\theta, z)$, являющейся результатом упругих столкновений электронов с атомами Не. Немонокинетичность ФРЭ в области энергий первоначального пучка авторы [1] описывали гауссовским распределением по энергии:

$$
\begin{aligned}
& T_{m}(E)=B \exp \left[-\frac{\left(E-E_{0}\right)^{2}}{\Delta_{t} E^{2}}\right], \\
& B=\frac{2}{\sqrt{\pi} \Delta_{T} E\left[1+\operatorname{erf}\left(\frac{E_{0}}{\Delta_{t} E}\right)\right]},
\end{aligned}
$$

где в условиях экспериментов авторов [1] $\Delta_{t} E \approx 1 \mathrm{eV}$, $E_{0}=30 \mathrm{eV}$ - средняя энергия электронов. Определенная таким образом ФРЭ $F_{s c}(\theta, z)$ при энергии электронов $30 \mathrm{eV}$ на различных расстояниях $z$ от катода 


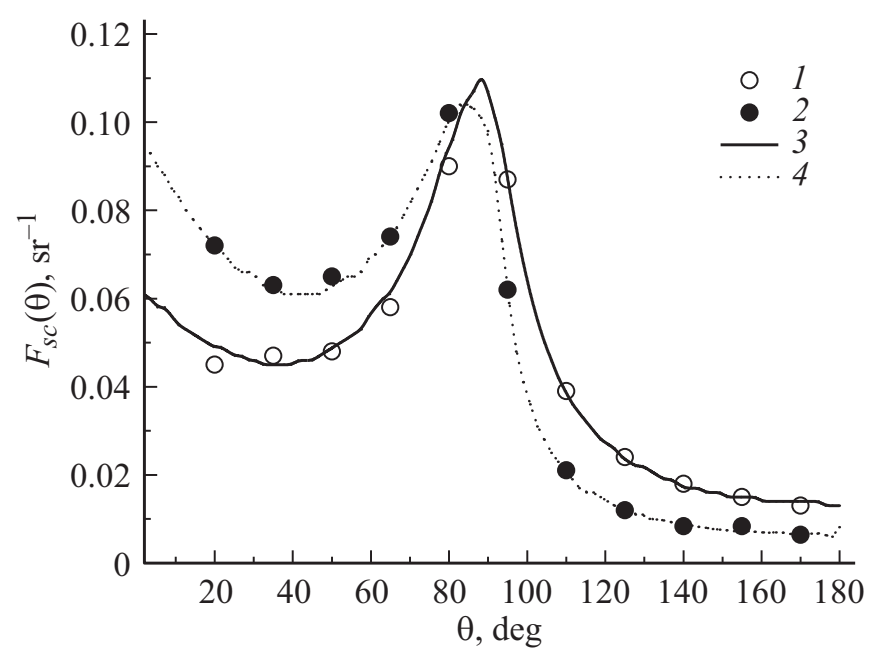

Рис. 1. Сравнение рассчитанной рассеянной части ФРЭ $F_{s c}(\theta, z)$ и экспериментально измеренной в НПР для следующих условий: $E_{0}=30 \mathrm{eV} ; \Delta_{t} E=1 \mathrm{eV} ; \Delta E=0.2 \mathrm{eV}$; $P=0.25$ Torr; $i=100 \mathrm{~mA} ; d=3 \mathrm{~mm}$; эксперимент, $z: 1-1$, $2-2 \mathrm{~mm}$; расчет, $z: 3-1,4-2 \mathrm{~mm}$.

приведена на рис. 1. Видно, что ее угловая зависимость имеет нетривиальный вид. Поскольку расстояние между электродами $d$ в условиях экспериментов [1], где исследовался пролетный режим, существенно меньше, чем длина пробега электрона относительно упругого рассеяния $\lambda_{e}$, то невелико и число упругих столкновений, которые испытывает электрон до того, как достигнет анода. Это позволяет надеяться, что можно, используя угловую зависимость $F_{s c}(\theta, z)$, найти индикатрису упругого рассеяния электрона на атоме плазмообразующего газа. Этому и посвящена настоящая работа.

При расчетах методом Монте-Карло нерассеянная часть ФРЭ нормирована на множитель $\exp \left(-\frac{z}{\lambda_{e}}\right)$. Экспериментально измеренная ФРЭ приведена к одной концентрации с рассчитанной. Данные взяты из работы [1].

\section{1. Получение основных соотношений}

\section{1. Аналитическое решение уравнения Больцмана для ФРЭ в пролетном режиме НПР}

Для того чтобы способ получения информации об индикатрисе рассеяния из ФР упруго рассеянных электронов $F_{s c}(\theta, z)$ в пролетном режиме НПР был эффективен, сама ФРЭ должна быть к ней чувствительна. На рис. 2 приведены результаты расчета $F_{s c}(\theta, z)$ методом МонтеКарло для индикатрисы рассеяния (нормированной на единицу), которая описывает экспериментальные данные [19] по упругому рассеянию электрона на атоме Непри энергии относительного движения электрона и атома $30 \mathrm{eV}$ :

$$
\bar{G}_{e a}\left(\theta_{0}\right)=0.378 \exp \left[-\left(\frac{6 \theta_{0}}{\pi}\right)^{1.3}\right]+0.0478
$$

где $\theta_{0}-$ угол рассеяния в радианахи изотропной индикатрисы рассеяния, равной $\frac{1}{4 \pi}$.

Видно, что ФР упруго рассеянных электронов для данных индикатрис существенно отличаются. Особенно велико отличие для углов $\theta \leq 90^{\circ}$.

Для нахождения связи между ФР упруго рассеянных электронов и индикатрисой рассеяния построим аналитическое решение уравнения Больцмана для рассматриваемого случая. Напомним основные положения физической модели формирования ФРЭ в области энергии первоначального пучка, сформулированные в работе [1], имея ввиду, что разряд инициируется в цилиндре диаметром $14 \mathrm{~mm}$ с металлическими стенками, которые находятся под потенциалом катода.При этом электроды имеют круговую форму и диаметр $11 \mathrm{~mm}$. Как уже отмечалось, в пролетном режиме НПР в инертных газах электрическое поле значительно только в катодном слое, толщина которого много меньше межэлектродного промежутка [20].

Введем декартовую систему координат, в которой ось $Z$ направлена от катода к аноду вдоль оси симметрии разряда. Из плоскости $z=0$, совпадающей с плоскостью катода, вдоль положительного направления оси $Z$ вылетает пучок электронов с начальной энергией $E_{0} \approx 30 \mathrm{eV}$. Плоскость вылета для электронов является полностью отражающей поверхностью. При $z=d$ находится полностью поглощающая плоскость (анод), при этом электрическое поле в пространстве между

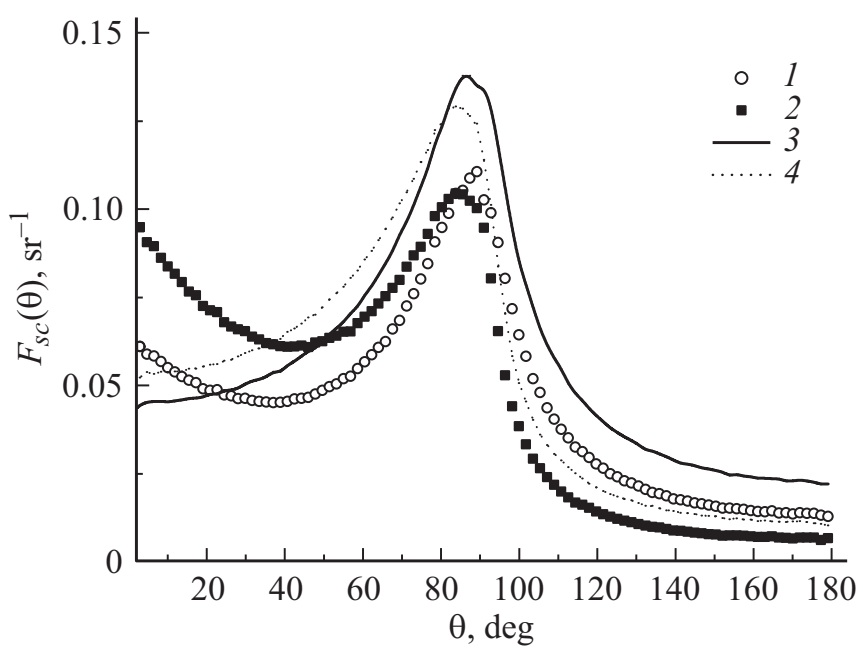

Рис. 2. ФРЭ $F_{s c}(\theta, z)$, рассчитанная методом Монте-Карло для условий рис. 1 при различных расстояниях от катода и для разных индикатрис упругого рассеяния электрона на атоме; $1-z=1 \mathrm{~mm}$, индикатриса $\bar{G}_{e a}\left(\theta_{0}\right) ; 2-z=2 \mathrm{~mm}$, индикатриса $\bar{G}_{e a}\left(\theta_{0}\right) ; 3-z=1 \mathrm{~mm}$, индикатриса $\frac{1}{4 \pi} ; 4-$ $z=1 \mathrm{~mm}$, индикатриса $\frac{1}{4 \pi}$. 
плоскостями отсутствует. При движении электроны испытывают упругие столкновения с атомами Не, которые происходят без потери их энергии. Здесь мы использовали приближение, не учитывающее наличие отражающих стенок в конструкции устройства, реализующего НПР. Легко показать, что решение поставленной задачи с учетом полностью отражающих боковых цилиндрических стенок в точности равно соответствующей ФРЭ для неограниченной области между двумя плоскостями.

В рамках сформулированной модели уравнение Больцмана для ФРЭ $F(\mu, \bar{z})$ имеет вид

$$
\mu \frac{\partial F}{\partial \bar{z}}+F=\int_{-1}^{1} g_{e}\left(\mu^{\prime} \rightarrow \mu\right) F\left(\mu^{\prime}, \bar{z}\right) d \mu^{\prime}
$$

с граничными условиями

$$
F(\mu, 0)=F(-\mu, 0), \quad F(\mu, \kappa)_{\mu<0}=0,
$$

где $\kappa=\Sigma_{e} d, \bar{z}=\Sigma_{e} z, \Sigma_{e}=\frac{1}{\lambda_{e}}-$ обратная длина свободного пробега электрона относительно упругих столкновений, $\mu^{\prime}, \mu-$ косинусы полярных углов, которые составляют скорость электрона с осью $Z$ до и после столкновения соответственно:

$$
g_{e}\left(\mu^{\prime} \rightarrow \mu\right)=\int_{0}^{2 \pi} \bar{G}_{e}\left[\mu_{0}\left(\mu^{\prime}, \mu, \varphi\right)\right] d \varphi,
$$

$\mu_{0}\left(\mu^{\prime}, \mu, \varphi\right)=\mu^{\prime} \mu+\sqrt{1-\mu^{\prime 2}} \sqrt{1-\mu^{2}} \cos \varphi, \mu_{0}$ - косинус угла упругого рассеяния электрона, - разница азимутальных углов скоростей электрона до и после столкновения, $\bar{G}_{e}\left[\mu_{0}\left(\mu^{\prime}, \mu, \varphi\right)\right]-$ индикатриса упругого рассеяния электрона на атоме Не, удовлетворяющая условию нормировки:

$$
\begin{gathered}
\int_{0}^{2 \pi} \int_{-1}^{1} \bar{G}_{e}\left[\mu_{0}\left(\mu^{\prime}, \mu, \varphi\right)\right] d \mu d \varphi=1, \\
\bar{G}_{e}\left[\mu_{0}\left(\mu^{\prime}, \mu, \varphi\right)\right]=\bar{G}_{e a}\left(\arccos \mu_{0}\right) .
\end{gathered}
$$

Первое из равенств (5) означает полное отражение электронов от плоского катода, второе - их поглощение плоским анодом.

Решение задач (4), (5) можно получить методом разложения ФРЭ по числам столкновений [21]. Учитывая, что величина $\kappa \leq 1$, можно ожидать, что ФРЭ, испытавших не более трех-четырех столкновений, будет хорошим приближением. Суть метода заключается в следующем. Очевидно, что ФРЭ $f_{n}(\mu, \bar{z})$, испытавших пстолкновений, формируется за счет одного столкновения электронов, испытавших $n-1$ столкновение. При этом частицы „уходят “ из числа формирующих $f_{n}(\mu, \bar{z})$ за счет $n+1$ столкновения. Таким образом, для определения $f_{n}(\mu, \bar{z})$ имеем систему рекуррентных уравнений

$$
\begin{gathered}
\mu \frac{\partial f_{n}}{\partial \bar{z}}+f_{n}=\int_{-1}^{1} g_{e}\left(\mu^{\prime} \rightarrow \mu\right) f_{n-1}\left(\mu^{\prime}, \bar{z}\right) d \mu^{\prime} \text { при } n>0 \\
f_{n}(\mu, 0)=f_{n}(-\mu, 0) ; \quad f_{n}(\mu, \kappa)_{\mu<0}=0, \\
\mu \frac{\partial f_{0}}{\partial \bar{z}}+f_{0}=0 ; \quad f_{0}(\mu, 0)=\frac{1}{2 \pi} \delta(\mu-1), \\
F(\mu, z)=\sum_{k=0}^{\infty} f_{k}(\mu \bar{z})=\sum_{k=0}^{i} f_{k}(\mu, \bar{z})+R_{f_{i+1}} \\
=f_{0}(\mu, \bar{z})+F_{s c}(\mu, \bar{z}),
\end{gathered}
$$

где $R_{f_{i+1}}-\Phi$ электронов, испытавших более чем $i$ столкновений.

Здесь мы заменили в граничном условии при $\bar{z}=0$ функцию $f^{0}(\mu, 0)$ на $\frac{1}{2 \pi} \delta(\mu-1)$, поскольку при величине параметра $\beta_{0} \approx 0.0017\left(T_{k}=0.1 \mathrm{eV} ; E_{0}=30 \mathrm{eV}\right)$, которая типична для рассматриваемого случая, они, очевидно, близки.

Уравнение (8) для $f_{n}(\mu, \bar{z})$ является линейным дифференциальным уравнением первого порядка и имеет аналитическое решение [22]:

$$
\begin{gathered}
\text { при } \mu<0, \quad n \geq 1, \\
f_{n}(\mu, \bar{z})=-\frac{\exp \left(-\frac{\bar{z}}{\mu}\right)}{\mu} \int_{\bar{z}}^{\kappa} \int_{-1}^{1} f_{n-1}\left(\mu^{\prime}, x\right) g_{e}\left(\mu^{\prime} \rightarrow \mu\right) \\
\times \exp \left(\frac{x}{\mu}\right) d \mu^{\prime} d x, \quad \text { при } \mu>0, \quad n \geq 1, \\
f_{n}(\mu \bar{z})=\exp \left(-\frac{\bar{z}}{\mu}\right) f_{n}(-\mu, 0)+\frac{\exp \left(-\frac{\bar{z}}{\mu}\right)}{\mu} \\
\times \int_{0}^{\bar{z}} \int_{-1}^{1} f_{n-1}\left(\mu^{\prime}, x\right) g_{e}\left(\mu^{\prime} \rightarrow \mu\right) \exp \left(\frac{x}{\mu}\right) d \mu^{\prime} d x \\
f_{0}(\mu, \bar{z})=\frac{\exp (-\bar{z})}{2 \pi} \delta(\mu-1), \\
\bar{G}_{e}(\mu) \\
(1-\mu)
\end{gathered}
$$

при $\mu<0$;

$$
\begin{aligned}
& f_{1}(\mu, \bar{z})=\bar{G}_{e}(\mu) \frac{\left[\exp (-\bar{z})--\exp \left(-\frac{\bar{z}}{\mu}\right)\right]}{(1-\mu)} \\
& +\bar{G}_{e}(-\mu) \frac{\left[\exp \left(-\frac{\bar{z}}{\mu}\right)-\exp \left(-\kappa-\frac{1}{\mu}(\kappa+\bar{z})\right)\right]}{(1+\mu)}
\end{aligned}
$$

при $\mu>0$. 
Интересно отметить, что в выражение для $f_{1}(\mu, \bar{z})$ в качестве множителя входит индикатриса упругого рассеяния электрона на атоме $\bar{G}_{e}(\mu)$. То есть, выбирая параметр $\kappa$ не слишком большим, так, чтобы роль столкновений с $n \geq 2$ была невелика, можно попытаться, исключая из измеренной зондовым методом ФРЭ [1] первоначальный пучок $f_{0}(\mu, \bar{z})$, оценить индикатрису рассеяния электрона на атоме. Ниже мы подробнее рассмотрим эту возможность.

\section{2. Способ оценки индикатрисы упругого рассеяния электрона на атоме из экспериментальных данных о ФРЭ в пролетном режиме НПР}

Предположим, что у нас есть экспериментально измеренная $\Phi \mathrm{P} F_{s c}(\mu, \bar{z})$ при некоторой энергии электронов $E_{0}$, т. е. с некоторым шагом по координате, определена ее угловая зависимость. Требуется из этих данных найти индикатрису рассеяния. Для решения данной задачи воспользуемся формулами (9) и перепишем решение для рассеянной части уравнения Больцмана (4) в виде:

$$
F_{s c}(\mu, \bar{z})=F_{1}(\mu, \bar{z})+\sum_{k=1}^{\infty} \hat{P}^{(k)}\left[F_{1}(\mu, \bar{z})\right],
$$

где

$$
\begin{array}{r}
F_{1}(\mu, \bar{z})=\bar{G}_{e}(\mu) \varphi_{1}(\mu, \bar{z}) \quad \text { при } \quad \mu<0, \\
F_{1}(\mu, \bar{z})=\bar{G}_{e}(\mu) \varphi_{2}(\mu, \bar{z})+\bar{G}_{e}(-\mu) \varphi_{3}(\mu, \bar{z})
\end{array}
$$

при $\mu>0$,

$$
\varphi_{1}(0, \bar{z})=\varphi_{2}(0, \bar{z})+\varphi_{3}(0, \bar{z}),
$$

а интегральный оператор $\hat{P}[F]$ и функции $\varphi_{1}(\mu, \bar{z})$, $\varphi_{2}(\mu, \bar{z}), \varphi_{3}(\mu, \bar{z})$, определяются очевидным образом из соотношения (9). Ясно, что ряд в формуле (10), представляющий решение уравнения Больцмана в виде разложения по числам столкновения, сходится, если уравнение Больцмана имеет единственное решение (т.е. в том числе и в рассматриваемом случае). Это можно доказать строго, используя то, что норма оператора $\|\hat{P}[F]\|<1$, где $F(\mu, \bar{z})$ - непрерывная (вместе с первой производной) функция своих аргументов, удовлетворяющая условию

$$
2 \pi \int_{-1}^{1} F(\mu, \bar{z}) d \mu \leq 1 .
$$

Будем считать, что соотношение (10) является уравнением для нахождения ФР частиц, испытавших одно столкновение $F_{1}(\mu, \bar{z})$. Решать уравнение (10) будем методом последовательных приближений

$$
\begin{gathered}
F_{1}(\mu, \bar{z})^{(i)}=F_{s c}(\mu, \bar{z})-\sum_{k=1}^{\infty} \hat{P}^{(k)}\left[F_{1}(\mu, \bar{z})^{(i-1)}\right], \\
F_{1}(\mu, \bar{z})^{(0)}=F_{s c}(\mu, \bar{z}) .
\end{gathered}
$$

Покажем, что решение $F_{1}(\mu, \bar{z})^{(i)}$ при $i \rightarrow \infty$ сходится к точному выражению $F_{1}(\mu, \bar{z})$, а сходимость данного метода превосходит сходимость ряда для ФР по числам столкновений в формуле (8). Действительно, действуя, согласно (12), с использованием (10) получаем

$$
\begin{gathered}
F_{1}(\mu, \bar{z})^{(0)}=F_{s c}(\mu, \bar{z})=F_{1}(\mu, \bar{z})+\sum_{k=1}^{\infty} \hat{P}^{(k)}\left[F_{1}(\mu, \bar{z})^{(i-1)}\right], \\
\left.F_{1}(\mu, \bar{z})^{(1)}=F_{1}(\mu, \bar{z})-\sum_{k_{1}=1}^{\infty} \hat{P}^{\left(k_{1}\right)}\left[\sum_{k_{2}=1}\right)^{\infty} \hat{P}^{\left(k_{2}\right)}\left[F_{1}(\mu, \bar{z})\right]\right], \\
F_{1}(\mu, \bar{z})^{(2)}=F_{1}(\mu, \bar{z})+\sum_{\left(k_{1}=1\right)}^{\infty} \hat{P}^{\left(k_{1}\right)} \\
\times\left[\sum_{k_{2}=1}^{\infty} \hat{P}^{\left(k_{2}\right)}\left[\sum_{k_{3}=1}^{\infty} \hat{P}^{\left(k_{3}\right)}\left[F_{1}(\mu, \bar{z})\right]\right],\right. \\
F_{1}(\mu, \bar{z})^{(i)}=F_{1}(\mu, \bar{z})+(-1)^{i} \sum_{k_{1}=1}^{\infty} \hat{P}^{\left(k_{1}\right)} \\
\times\left[\sum_{k_{2}=1}^{\infty} \hat{P}^{\left(k_{2}\right)}\left[\ldots \hat{P}^{k_{(i)}} \sum_{k_{i+1}=1}^{\infty} \hat{P}^{\left(k_{i+1}\right)}\left[F_{1}(\mu, \bar{z})\right] \ldots\right]\right. \\
F_{2}(\mu, \bar{z})+(-1)^{i} \sum_{k_{1}=1}^{\infty} \ldots \sum_{k_{i+1}=1}^{\infty} \hat{P}^{\left(\Sigma_{i=1}^{i}=k_{j}\right)}\left[F_{1}(\mu, \bar{z})\right] .
\end{gathered}
$$

Из (13) при $i \rightarrow \infty$ имеем

$$
\begin{aligned}
& F_{1}(\mu, \bar{z})=F_{s c}(\mu, \bar{z})+\sum_{i=1}^{\infty}(-1)^{i} \sum_{k=1}^{\infty} \hat{P}^{(k)} \\
& \times\left[\sum_{k=1}^{\infty} \hat{P}^{(k)}\left[\ldots \hat{P}^{(k)} \sum_{k+1}^{\infty} \hat{P}^{(k)}\left[F_{s c}(\mu, \bar{z})\right]\right],\right.
\end{aligned}
$$

где в $i$-том члене ряда (14) количество „вложенных“ аргументов оператора $\hat{P}^{(k)}$ равно $i$.

Таким образом, получаем, что старший член ряда в правой части соотношения $(13)$ для $F_{1}(\mu, \bar{z})^{(i)}$ имеет вид $\hat{P}^{(i+1)}\left[F_{1}(\mu, \bar{z})\right]$ - такой же, как $(i+1)$-й член в сходящемся ряду в правой части выражения (10). Отсюда следует, что при $i \rightarrow \infty$ ряд в правой части (13) стремится к нулю, а $\lim _{i \rightarrow \infty} F_{1}(\mu, \bar{z})^{(i)}=F_{1}(\mu, \bar{z})$.

Таким образом, предложенный метод последовательных приближений сходится к точному решению $F_{1}(\mu, \bar{z})$. Нетрудно показать, используя явный вид оператора $\hat{P}[F]$, что, поскольку ФР электронов, испытавших любое число столкновений, положительна в области определения, то выражение $\hat{P}\left[F_{s c}(\mu, \bar{z})\right]>0$ при любых $-1 \leq \mu \leq 1$; $0 \leq \bar{z} \leq \kappa$. Но тогда ряд (14) является знакопеременным и при увеличении $i$ убывает быстрее, чем остаток ряда $R_{f i+1}$ в соотношении (8), а относительная ошибка приближения определяется относительным вкладом в ФР электронов, испытавших $i+1$ столкновение. 
Из соотношений (13) также следует, что для четных и нечетных приближений выполняется

$$
\begin{gathered}
F_{1}(\mu, \bar{z})^{(2 i)}<F_{1}(\mu, \bar{z}), \quad F_{1}(\mu, \bar{z})^{(2 i+1)}>F_{1}(\mu, \bar{z}), \\
i=0,1, \ldots
\end{gathered}
$$

Но тогда выполняется неравенство

$$
\begin{aligned}
& \left|\bar{F}_{1}(\mu, \bar{z})^{(i)}-F_{1}(\mu, \bar{z})\right|=\mid 0.5\left[F_{1}(\mu, \bar{z})^{(i)}+F_{1}(\mu, \bar{z})^{(i+1)}\right] \\
& -F_{1}(\mu, \bar{z})|<0.5| F_{1}(\mu, \bar{z})^{(i)}-F_{1}(\mu, \bar{z}) \mid \\
& <0.5\left|F_{1}(\mu, \bar{z})^{(i+1)}-F_{1}(\mu, \bar{z})\right| .
\end{aligned}
$$

Действительно,

$$
\begin{aligned}
& 0.5\left[F_{1}(\mu, \bar{z})^{(i)}+F_{1}(\mu, \bar{z})^{(i+1)}\right]-F_{1}(\mu, \bar{z})=0.5 \\
& \times\left[F_{1}(\mu, \bar{z})^{(i)}-F_{1}(\mu, \bar{z})\right]+0.5\left[F_{1}(\mu, \bar{z})^{(i+1)}-F_{1}(\mu, \bar{z})\right] .
\end{aligned}
$$

Как было показано выше, при любом $i$ слагаемые в последнем соотношении имеют разный знак (см. неравенства (15)), откуда и следует (16).

Таким образом, при любом заданном значении $i$ наименьшее отклонение от точного решения имеет функция

$$
\bar{F}_{1}(\mu, \bar{z})^{i}=0.5\left[F_{1}(\mu, \bar{z})^{(i)}+F_{1}(\mu, \bar{z})^{(i+1)}\right],
$$

и это отклонение не менее чем в два раза меньше отклонения от точного решения приближений $F_{1}(\mu, \bar{z})^{(i)}$ и $F_{1}(\mu, \bar{z})^{(i+1)}$ (см. неравенства (16)).

При $\mu<0$ индикатриса рассеяния связана с ФР частиц, испытавших одно столкновение, алгебраически, что позволяет из $F_{1}(\mu, \bar{z})^{(i)}$ найти $G_{e}(\mu)^{(i)}$. Индикатриса рассеяния при $\mu>0$ находится совершенно аналогично с использованием найденной $G_{e}(\mu)^{(i)}$ при $\mu<0$.

Таким образом, зная точную часть ФР электронов, испытавших не менее одного столкновения, можно при условии достаточно быстрой сходимости ряда (10) найти индикатрису рассеяния $\bar{G}_{e}\left(\mu_{0}\right)$. Учитывая вышесказанное, наилучшее совпадение при минимальном значении индекса $i$ получим, если возьмем в качестве индикатрисы рассеяния величину

$$
G_{e i}(\mu)=0.5\left[G_{e}(\mu)^{(i)}+G_{e}(\mu)^{(i+1)}\right] .
$$

С использованием формулы (11) для величины $G_{e i}(\mu)$ имеем

$$
G_{e i}(\mu)=0.5\left[F_{1}(\mu, \bar{z})^{(i)}+F_{1}(\mu, \bar{z})^{(i+1)}\right] / \varphi_{1}(\mu, \bar{z})
$$

при $\mu<0$,

$$
\begin{aligned}
& G_{e i}(\mu)=\left\{0.5\left[F_{1}(\mu, \bar{z})^{(i)}+F_{1}(\mu, \bar{z})^{(i+1)}\right]\right. \\
& \left.-\frac{0.5\left[F_{1}(-\mu, \bar{z})^{(i)}+F_{1}(-\mu, \bar{z})^{(i+1)}\right] \varphi_{3}(\mu, \bar{z})}{\varphi_{1}(-\mu, \bar{z})}\right\} / \varphi_{2}(\mu, \bar{z})
\end{aligned}
$$

при $\mu>0$
При этом для определения $F_{1}(\mu, \bar{z})^{(i)}$ в формуле (12) можно использовать либо результаты численных расчетов методом Монте-Карло, либо экспериментально определенную ФР рассеянных электронов. Ниже мы покажем результаты применения разработанной методики.

\section{2. Обсуждение полученных результатов}

Для проверки правильности разработанной аналитической теории мы сравнили результаты расчетов по формулам (9) рассеянной части ФРИ для $E_{0}=30 \mathrm{eV} \mathrm{c}$ аналогичными данными, полученными методом МонтеКарло с использованием индикатрисы рассеяния, определенной соотношением (10). При аналитических расчетах учитывалось три столкновения. Результаты приведены на рис. 3. Видно, что при $\kappa=0.3$ наблюдается хорошее соответствие аналитических и численных расчетов для $\bar{z}=\frac{1}{3}, \frac{2}{3}$, как для рассеяния „вперед“, так и для рассеяния „назад“. В тоже время наблюдается небольшая разница при углах, близких к $\theta=90^{\circ}$. Это вполне очевидный результат, поскольку из-за полностью отражающих стенок (и диаметре объема плазмы порядка межэлектродного расстояния) эффективный путь, который проходят электроны в поперечном направлении, заметно больше, чем расстояние между электродами. Строго говоря, электроны, которые движутся точно поперек оси разряда, проходят бесконечное расстояние, отражаясь от стенок. Поэтому для них приближение, когда учитываются частицы, испытавшие не более трех столкновений, при данном значении параметра $\kappa$ описывает реальную ФР несколько хуже, чем для электронов, движущихся в других направлениях.

Обсудим теперь результаты по восстановлению индикатрисы рассеяния. Отметим, что в формулах (10)-(14) суммирование производится до бесконечности, что, как следует из физического смысла решения (10), означает учет бесконечного числа упругих столкновений (в данном случае электрона с атомом Не). В нашем случае, как видно из данных рис. 3, при значении параметра $\kappa=0.3$ достаточно провести учет трех столкновений. При этом вклад третьего столкновения (член $\hat{P}^{(2)}\left[F_{1}(\mu, \bar{z})\right]$ в формуле $\left.(10)\right)$ составляет менее $10 \%$. Но тогда можно ожидать, что также мало будет отклонение индикатрисы, найденной по соотношению (18),от точной при $i=0$ из данных о рассеянной части ФРЭ при $\kappa=0.3$. При параметре $\kappa>0.3$, по-видимому, будет необходим учет четвертого и более столкновений. Кроме того, как показано в работе [1], из-за того, что ФР электронов, не испытавших столкновений, вносит заметный вклад во вторую производную зондового тока при углах, когда нормаль к проводящей поверхности зонда составляет угол с направлением электрического поля в плазме меньше $20^{\circ}$, экспериментальное определение рассеянной части ФРЭ не представляется возможным. Как следствие, и восстановление индикатрисы упругого 


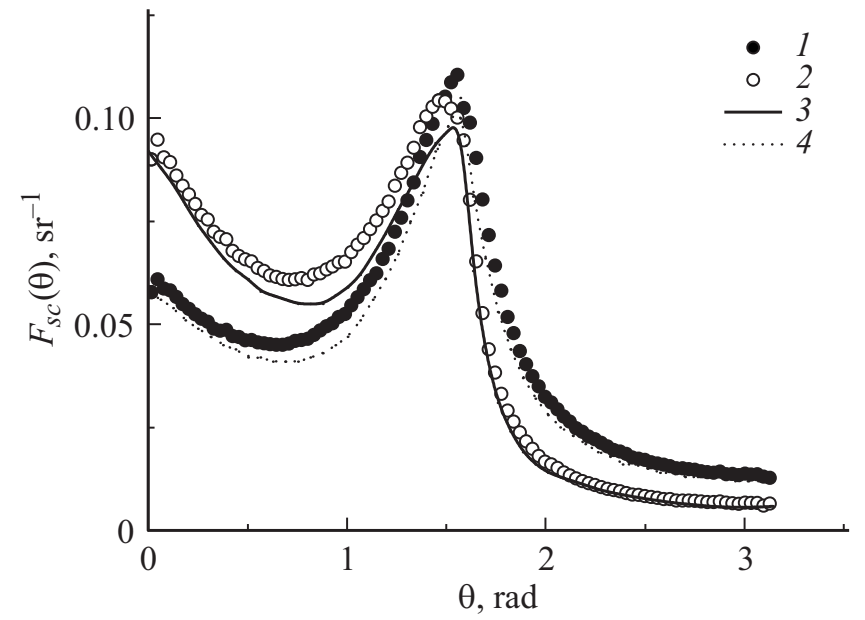

Рис. 3. Сравнение расчетов рассеянной части ФРЭ в Не по методу Монте-Карло и вычисленной по формулам (9) с учетом первых трех столкновений для отношения расстояния между электродами к длине пробега $\kappa=0.3$ на различных относительных расстояниях от катода; $E_{0}=30 \mathrm{eV} ; 1,2$ расчет методом Монте-Карло при $\bar{z}=\frac{1}{3}, \frac{2}{3}$ соответственно; 3,4 - аналитический расчет с учетом трех столкновений при $\bar{z}=\frac{2}{3}, \frac{1}{3}$ соответственно.

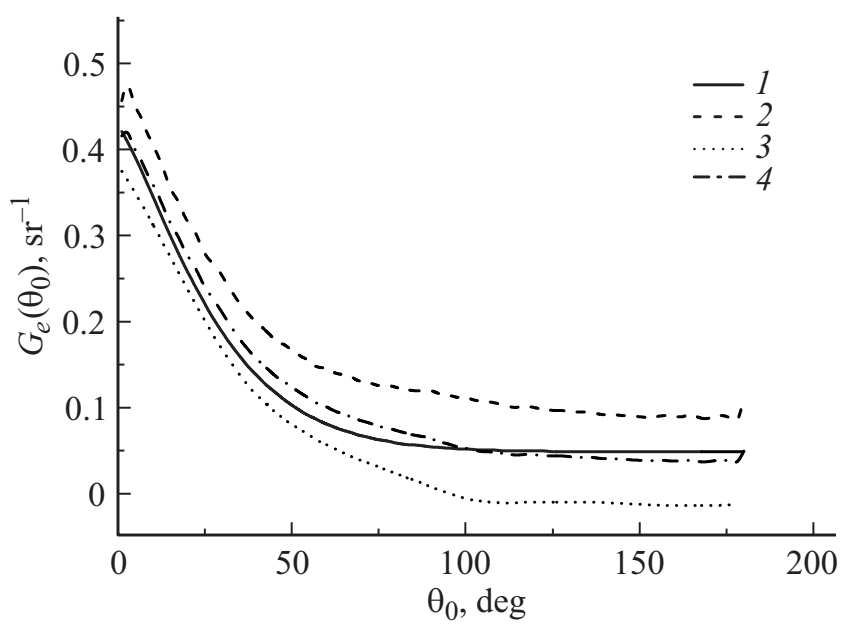

Рис. 4. Точная индикатриса упругого рассеяния электрона на атоме Не $\bar{G}_{e}(1)$; нулевое приближение индикатрисы $G_{e}^{(0)}(2)$ и первое приближение $G_{e}^{(1)}(3) ; 4-$ индикатриса $G_{e 0}$, рассчитанная по формуле (18) при $i=0 ; \kappa=0.3 ; \bar{z}=\frac{2}{3}$.

рассеяния электрона затруднено в указанном диапазоне углов рассеяния.

На рис. 4 приведены результаты сравнения $G_{e 0}(\mu)$ восстановленной из точной ФР при $\kappa=0.3, \bar{z}=2 / 3$, рассчитанной методом Монте-Карло, и точной индикатрисы $\bar{G}_{e}(\mu)$. Приведенные данные полностью подтверждают сделанные ранее выводы о том, что оптимальное приближение индикатрисы упругого рассеяния при минимальном значении $i$ определяется формулой (18). Видно, что различие восстановленной и точной индикатрис упругого рассеяния невелико и можно, таким образом, констатировать, что предложенная методика в условиях НПР позволяет восстановить из рассеянной части ФРЭ индикатрису упругого рассеяния при столкновении с атомом плазмообразующего газа.

На рис. 5 приведены вычисленные по формуле (18) из данных о ФРЭ при различных расстояниях $\bar{z}=2 / 3$, $1 / 3$ при $\kappa=0.3$ индикатрисы рассеяния $G_{e 0}$. Видно, что различие между ними пренебрежимо мало (существенно меньше, чем различие между известными данными [19,23]), хотя ФРЭ, рассчитанные методом Монте-Карло при $\bar{z}=2 / 3,1 / 3$, значительно различаются (рис. 3).Эти результаты свидетельствуют об эффективности предложенного способа восстановления индикатрисы упругого рассеяния электрона на атоме из данных о рассеянной части ФРЭ.

На этом же рисунке приведены результаты восстановления по вышеописанной процедуре индикатрисы рассеяния электрона на атоме Не при энергии электрона $30 \mathrm{eV}$ из рассеянной части ФРЭ при энергии электронов $30 \mathrm{eV}$, экспериментально измеренной по методике, описанной в работе [1]. Процедура восстановления проходила в два этапа: на первом — восстанавливалась рассеянная часть ФРЭ, а затем по формулам (18) - сама индикатриса. При зондовых измерениях мы пользовались методиками и аппаратурой, подробно описанными в [1,15-17]. При вычислении интегралов в (18) экспериментальные данные о $F_{s c}$ в области углов более $20^{\circ}$ аппроксимировались кубическими сплайнами, а менее $20^{\circ}$ - прямой, которая сшивалась с $F_{s c}(\theta)_{\theta} \rightarrow+20^{\circ}$. Из приведенных на рис. 5 (точки) данных следует,

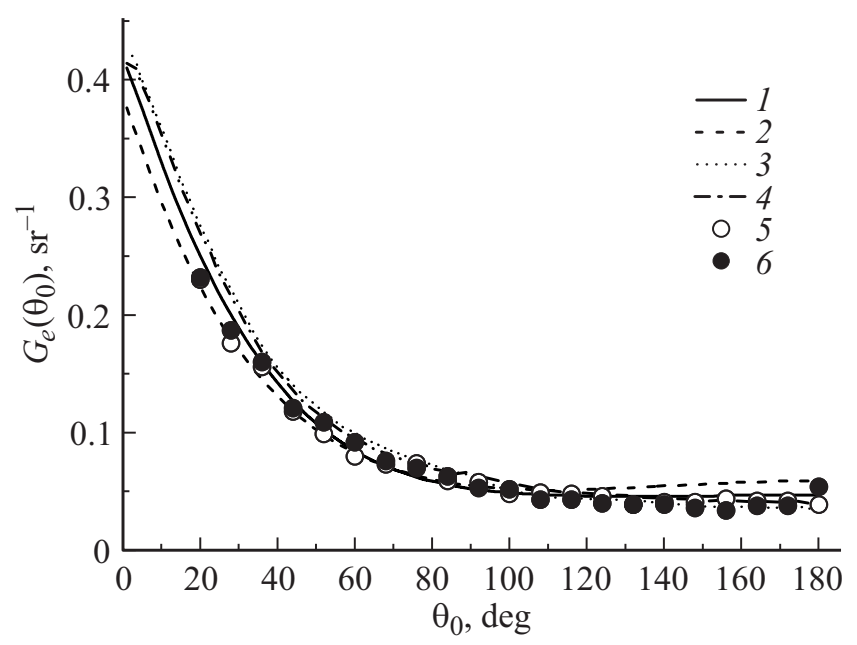

Рис. 5. Индикатриса упругого рассеяния электрона на атоме Не при энергии относительного движения $30 \mathrm{eV}$; 1 - данные [19]; 2 - данные [23]; 3,4 - восстановление по разработанной методике из рассчитанной методом Монте-Карло ФР рассеянных электронов с использованием индикатрисы (3) при $\bar{z}=\frac{1}{3}, \frac{2}{3}$ соответственно; 5,6 - восстановление по разработанной методике из экспериментальных данных о ФР рассеянных электронов [1] при $\bar{z}=\frac{1}{3}, \frac{2}{3}$ соответственно; $\kappa=0.3$. 
чтоиндикатрисы упругого рассеяния электрона на атоме Не, восстановленные из экспериментально измеренной ФРЭ при различных расстояниях до катода, отличаются незначительно и достаточно близки к приведенным в $[19,23]$. Тем не менее нельзя однозначно сказать какие экспериментальные данные из $[19,23]$ предпочтительнее. Для рассеяния „назад“ полученная нами индикатриса ближе к значениям, приведенным в [19], в то время как для рассеяния „вперед“ наши данные лежат между значениями, которые дают авторы $[19,23]$.

Как указывалось ранее, в данных условиях затруднительно получение информации по описанной выше методике об индикатрисе рассеяния при углах менее $20^{\circ}$. Однако поскольку, как известно, дифференциальное сечение упругого рассеяния электрона на атоме в этой области монотонно зависит от угла рассеяния, возможна экстраполяция полученных данных в область малых углов рассеяния.

\section{Заключение}

В заключение отметим, что для реализации разработанной методики определения индикатрисы упругого рассеяния электрона на атоме не требуется никаких априорных данных. Условием применимости этой методики является применимость зондового метода определения анизотропной ФРЭ методом плоского одностороннего зонда для пучка электронов с узким, в известной степени, распределением по энергиям (см. [1]). Кроме того, необходимо обеспечить достаточно быструю сходимость решения уравнения Больцмана для рассеянных электронов методом разложения по числам столкновения к точному решению. При численных вычислениях разумное время расчетов удается обеспечить, учитывая не более пяти столкновений. Для этого, как показали многочисленные расчеты, достаточно выполнения соотношения $\kappa<0.6$.

Тот факт, что найденная индикатриса упругого рассеяния электрона на атоме не зависит от вида ФРЭ и согласуется с известным экспериментальными данными, свидетельствует об адекватности разработанной методики.

\section{Благодарности}

Авторы выражают глубокую признательность профессору В.И. Кузнецову за критические замечания и полезные обсуждения результатов работы.

\section{Список литературы}

[1] Сухомлинов В.С., Мустафаев А.С., Страхова А.А., Мурильо О. // ЖТФ. 2017. Т. 87. Вып. 12. С. 1824-1833.

[2] Федоров В.Л. // ЖТФ. 1985. Т. 55. Вып. 5. С. 926-929. [Fedorov V.L. // Tech. Phys. 1985. Vol. 30. N 5. P. 554.]

[3] Мезенцев А.П., Мустафаев А.С., Федоров В.Л. // ЖТФ. 1985. Т. 55. Вып. 3. С. 544-549. [Mezentsev A.P., Mustafaev A.S., Fedorov V.L. // Tech. Phys. 1985. Vol. 30. N 3. P. 322].

[4] Мезенщев А.П., Мустаббаев А.С. // ЖТФ. 1985. Т. 55. Вып. 11. C. 2232-2235. [Mezentsev A.P., Mustafaev A.S. // Tech. Phys. 1985. Vol. 30. N 11. P. 1319.]

[5] Мезениев А.П., Мустафаев А.С., Федоров В.Л. // ЖТФ. 1986. Т. 56. Вып. 11. С. 2104-2110. [Mezentsev A.P., Mustafaev A.S., Fedorov V.L. // Tech. Phys. 1986. Vol. 31. N 11. P. 1263.]

[6] Mezentsev A.P., Mustafaev A.S. // J. Phys. D. 1986. Vol. 19. P. L69.

[7] Mezentsev A.P., Mustafaev A.S., Lapshin V.F., Fedorov V.L. // J. Phys. B. 1987. Vol. 20. P. L723.

[8] Mezentsev A.P., Mustafaev A.S., Fedorov V.L. // J. Phys. D. 1988. Vol. 21. P. 1464.

[9] Федоров В.Л., Мезениев А.П. // ЖТФ. 1987. Т. 57. Вып. 3. C. 595-597. [Fedorov V.L., Mezentsev A.P. // Tech. Phys. 1987. Vol. 32. P. 363.]

[10] Лапшин В.Ф., Мустафбаев А.С. // ЖТФ. 1989. Вып. 2. C. 35-45. [Lapshin V.F., Mustafaev A.S. // Tech. Phys. 1989. Vol. 34. N 2. P. 150.$]$

[11] Klagge S., Lunk A. // J. Appl. Phys. 1991. Vol. 70. P. 99.

[12] Klagge S. // Plasma Chem. Plasma Process. 1992. Vol. 12. P. 103.

[13] Мальков М.А. // Физика плазмы. 1990. Т. 16. С. 467-471. [Mal'kov M.A. // J. Plasma Phys. 1990. 1B. P. 267.]

[14] Woods R.C., Sudit I.D. // Phys. Rev. E. 1994. Vol. 50. N 3. P. 2222-2228.

[15] Мустафаев А.С., Сухомлинов В.С., Аинов М.А. // ЖТФ. 2015. Т. 85. Вып. 12. С. 45-55. [Mustafaev A.S., Sukhomlinov V.S., Ainov M.A. // Tech. Phys. 2015. Vol. 60. N 12. P. 1778-1789.]

[16] Mustafaev A.S., Sukhomlinov V.S. // J. Mining Institute. 2016. Vol. 222. P. 864-868.

[17] Сухомлинов В.С., Мустафаев А.С., Аинов М.А. // ТВТ. 2017. T. 55. № 3. C. 346-351.

[18] Сухомлинов В.С., Мустафаев А.С., Аинов М.А. // ТВТ. 2017. T. 55. № 4. C. 482-490.

[19] Adibzadeh M., Theodosiou C.E. // Atomic Data and Nuclear Data Tables. 2005. Vol. 91. P. 8-76.

[20] Мустафаев А.С. // ЖТФ. 2001. Т. 71. Вып. 4. С. 111-121.

[21] Марчук Г.И. // Методы расчета ядерных реакторов. М.: Гос. изд-во лит-ры в области атомной науки и техники, $1981.667 \mathrm{c}$.

[22] Корн Г., Корн Т. // Справочник по математике для научных работников и инженеров. М.: Наука, 1973. 832 с.

[23] Font W.C., Berrington K.A., Hibbert A. // J. Phys. B: At. Mol. Phys. 1981. Vol. 14. P. 307-321.

\section{Конфликт интересов}

Авторы заявляют, что у них нет конфликта интересов. 